\title{
Visibilidade no Facebook: Modelos, Medições e Implicações
}

\author{
Eduardo Hargreaves ${ }^{1}$, \\ Daniel Menasché ${ }^{1}$, Giovanni Neglia ${ }^{2}$, Claudio Agosti $^{3}$ \\ ${ }^{1}$ Dept. Ciência da Computação, UFRJ, Rio de Janeiro, Brasil \\ ${ }^{2}$ INRIA, Sophia Antipolis, França \\ ${ }^{3}$ Universidade de Amsterdã, Amsterdã, Holanda \\ eduardo@hargreaves.tech, sadoc@dcc.ufrj.br,giovanni.neglia@inria.fr \\ claudio.agosti@logioshermes.org
}

\begin{abstract}
Resumo. O Facebook tem um impacto significativo na vida de milhões de usuários da Internet, todos os dias. Entretanto, os mecanismos usados pelo Facebook para filtrar mensagens apresentadas aos usuários não são de domínio público, motivando uma engenharia reversa dos mesmos. Neste trabalho, propomos modelos e medições para melhor entender o comportamento de timelines. Em particular, reportamos resultados sobre medições de visibilidade de fontes das eleições italianas de 2018, que motivam um modelo analítico para caracterizar a visibilidade de posts. Dentre as implicações práticas de nossos estudos, indicamos seu potencial para inferir diferentes métricas de visibilidade a partir de medidas colhidas do sistema.
\end{abstract}

\begin{abstract}
Facebook news feed personalization algorithm has a significant impact, on a daily basis, on the lifestyle, mood and opinion of millions of Internet users. Nonetheless, such algorithms lack transparency challenging researchers to improve their fairness and accountability. In this paper, we propose a model to capture the dynamics of contents over a timeline (also known as news feed). The input to our model is a fundamental quantity associated to timelines, which we show that can be easily parameterized using real world data: the arrival rate of posts of a given publisher followed by the user. Using real world Facebook traces from the latest elections in Italy, we validate the accuracy of the proposed model and use the model for conterfactual what-if analysis.
\end{abstract}

\section{Introdução}

O Facebook tem um impacto significativo na vida de milhões de usuários da Internet, todos os dias [Tsaparas 2017]. Entretanto, os mecanismos usados pelo Facebook para filtrar mensagens apresentadas aos usuários não são de domínio público, motivando pesquisas que envolvem desde estatísticas básicas até engenharia reversa de algoritmos. Tais estudos são fundamentais para garantir certo nível de transparência aos usuários do sistema.

Compreender um sistema complexo como o Facebook não é trivial. A visibilidade de uma certa fonte, por exemplo, depende da taxa de publicação de postagens por parte da fonte e dos interesses dos usuários. No artigo publicado em [TechCruch 2016] é dito que o Facebook utiliza aproximadamente 100.000 variáveis para escolher a publicações exibidas na suas News Feeds. Uma abordagem para avaliar o impacto de diferentes fatores 
na visibilidade consiste na criação de modelos analíticos que permitam derivar métricas de interesse a partir de dados colhidos das redes, mas tais dados em geral não estão disponíveis de forma aberta.

Neste trabalho, propomos medições e um modelo para melhor entender o comportamento de timelines. O modelo proposto é baseado em modelos de filas e de caches, para os quais existem uma vasta literatura. Em particular, parametrizamos e validamos o modelo proposto usando dados do Facebook, indicando o poder expressivo do mesmo.

Contribuições Dentre as principais contribuições deste trabalho, destacamos as seguintes.

Medições do Facebook Usando usuários virtuais, colhemos visões distintas sobre as postagens no Facebook nas eleições da Itália em 2018. Tais medições motivam a criação de modelos para capturar a essência da dinâmica das publicações em timelines.

Modelo de visibilidade Propomos um modelo para estimar a visibilidade das publicações em função das taxas de criação das fontes e do algoritmo de filtragem da rede social. Usando dados reais do Facebook, validamos os modelos propostos.

Estudo contrafactual de caso Usando as medições e os modelos propostos, avaliamos qual teria sido a visibilidade de diferentes publicações, sob diferentes critérios de interesse.

O restante deste artigo está organizado da seguinte forma. Na Seção 2 apresentamos a metodologia de medições adotada neste trabalho. Em seguida, a Seção 3 traz os resultados empíricos obtidos nas eleições italianas. A Seção 4 traz uma visão geral sobre o modelo proposto, seguida pela Seção 5 que apresenta o modelo analíticos proposto. A Seção 6 faz uma análise contrafactual dos dados usando o modelo proposto, seguida da Seção 7 que cobre trabalhos relacionados. A Seção 8 conclui.

\section{Medições}

Obtenção das métricas de interesse Usando a infraestrutura do Facebook Tracking Exposed, ${ }^{1}$ criamos seis usuários virtuais no Facebook. Todos os usuários seguem as mesmas fontes. Entretanto, os usuários possuem diferentes perfis (e.g., um curte páginas de direita e outro de esquerda). A cada hora, colhemos as publicações apresentadas na timeline de cada usuários. Tais fotografias constituem nossa base de dados.

Cabe destacar que a API do Facebook desde 2015 não oferece os dados acima [Facebook 2018b]. Mesmo quando a API era aberta, o Facebook dizia que a informação fornecida pela API não era fidedigna [Facebook 2018a]. Utilizamos esta API somente para colhermos informações sobre o número de publicações por fonte.

Métricas de interesse Dentre as métricas de interesse, destacamos as seguintes:

Repercussão (probabilidade de acerto) de uma fonte é a probabilidade de um usuário efetivamente ler (e possivelmente clicar) em uma publicação. Nesse trabalho, não distinguimos entre probabilidade de acerto e probabilidade de click. A probabilidadde de acerto de uma fonte pode ser dada pela visibilidade ou pela ocupação das postagens desta fonte.

\footnotetext{
${ }^{1}$ Facebook tracking exposed: https: / facebook.tracking.exposed/
} 
Visibilidade é a probabilidade de existir uma publicação de uma determinada fonte na timeline.

Ocupação é o número de publicações de uma determinada fonte na timeline.

Denotamos por $\pi_{i j}$ a visibilidade da fonte $j$ na timeline do usuário $i$. Denotamos por $N_{i j}$ o número médio de publicações do publisher $j$ na timeline de $i$. Neste trabalho, assumimos que a repercussão de uma fonte pode ser dada em função da visibilidade ou da ocupação. A repercussão igual a visibilidade condiz com usuários que eventualmente irão ler uma das publicações de cada uma das fontes presentes nas $K$ primeiras posições de suas timelines. A repercussão igual a ocupação, por outro lado, captura o comportamento de usuários que irão influenciar-se mais por fontes que ocupam mais posições em suas timelines, ou seja, quanto mais posições ocupadas por uma fonte, maior a repercussão da mesma.

\section{Descobertas empíricas sobre a eleição italiana}

\subsection{Coleta dos dados}

As eleições italianas foram no dia 04 de março de 2018 e o experimento ocorreu entre os dias 10 de janeiro de 2018 e 06 de março de 2018 de forma que procuramos analisar o período antecedente às eleições e os dois dias seguintes para capturarmos as reações ao resultado das eleições.

Foram selecionadas 30 fontes italianas que posteriormente foram classificadas de acordo com uma das cinco orientações políticas: esquerda, centro-esquerda, direita, ultradireita e movimento 5 estrelas. Também foram criados 6 usuários fictícios. Todos os usuários seguem as mesmas 30 fontes. No entanto, os usuários foram polarizados de forma que cada usuário curtia publicações de apenas uma orientação política. O sexto usuário foi caraterizado como indeciso uma vez que não curtiu publicações de numa página.

As "fotografias" das timelines foram "tiradas"através de uma extensão dos navegadores Chrome e Firefox chamada facebook.tracking.exposed. Essa extensão coleta os dados públicos, e retorna a data de criação da publicação, o momento da visualização, o usuário que visualizou, a fonte, o conteúdo, a quantidade de reações, o número de compartilhamentos, e a ordem de aparição da publicação. Em paralelo, a API do Facebook foi utilizada para a obtenção de todas as publicações das fontes selecionadas. Chamamos de $S_{i}$ o número de fotografias tiradas no $i$-ésimo usuário. Nos nossos experimentos, os usuários foram indexados de 1 a 6 , denotando as orientações de centro-esquerda, ultradireita, esquerda, M5S, direita e a indecisa. Os valores de $S_{i}$ obtidos foram: 577, 504, $623,674,655,576, \operatorname{com} i=1, \ldots, 6$. A diferença entre o número de fotografias advém de falhas de medição, e.g., máquinas travarem, falta de luz e queda de conexão. Embora existam tais falhas, acreditamos que estatisticamente elas não afetam os resultados, tendo em vista o grande volume de fotografias colhidas.

\subsection{Achados empíricos}

A seguir, apresentamos uma visão geral dos dados colhidos. A Figura 1 (a) ilustra o número de publicações por fonte. Esta informação foi colhida diretamente da API do Facebook. Algumas poucas fontes geraram milhares de publicações durante o período considerado, enquanto que a maioria gerou dezenas de publicações. 


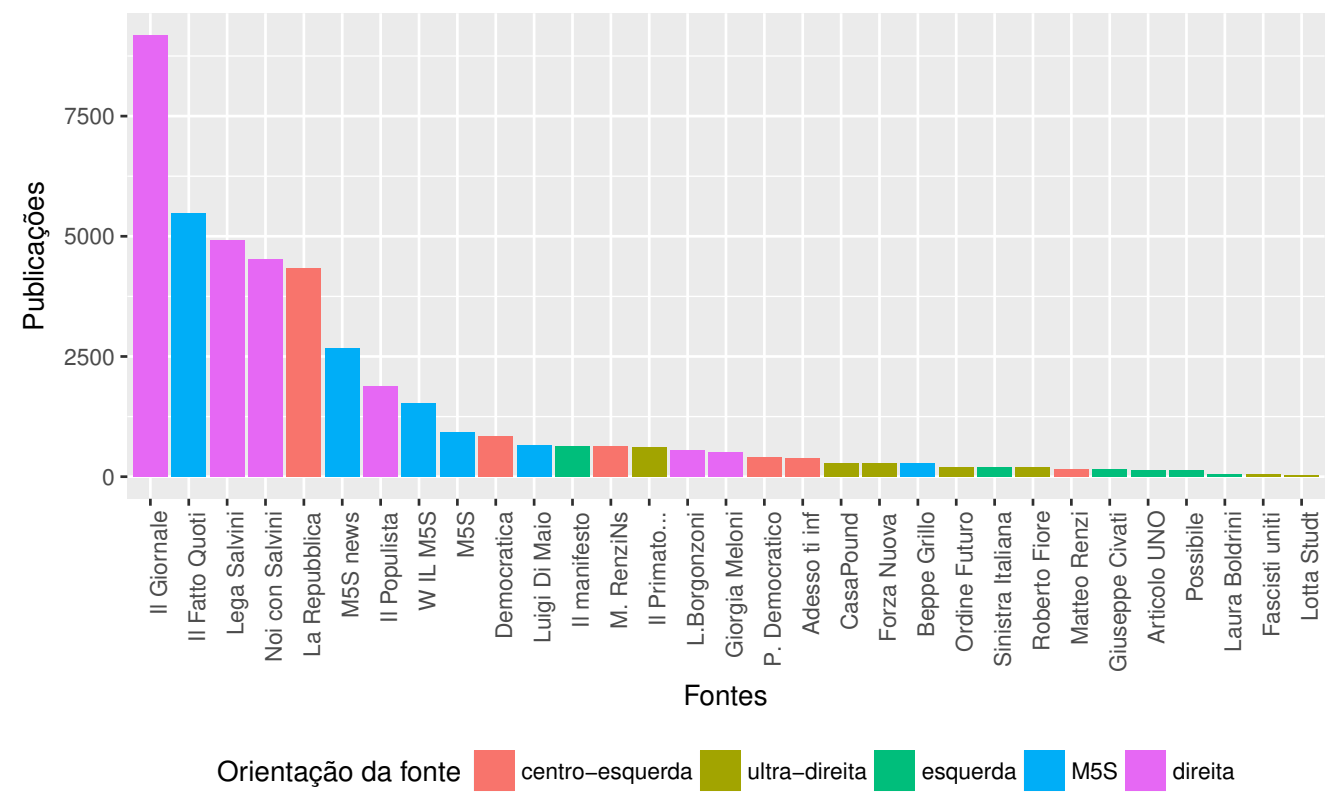

Figura 1. Total de publicações por fonte

Chamamos cada publicação visualizada de impressão, e chamamos de $I_{i j}$ o número total de impressões da fonte $j$ no usuário $i$. Se uma publicação é visualizada duas vezes, ela conta com duas impressões. Esta informação foi colhida a partir de nosso aplicativo (Facebook Tracking Exposed). A Figura 2 mostra o total de impressões $I_{i j}$ por usuário. Em nenhum usuário a distribuição das publicações vistas foi semelhante a distribuição das publicações criadas. Em todos os usuários, as fontes mais vistas refletiram a polarização dos mesmos. Por exemplo, o usuário que curtiu fontes de esquerda (no topo a direita da Figura 2) visualizou mais postagens de fontes de esquerda do que os demais. Cabe relembrar que todos os usuários seguiram todas as fontes (e apenas distinguiram-se pelas curtidas) e que o viés fica claro independente da taxa com quem as fontes geraram conteúdos (ilustrada na Figura 1).

A Figura 3 (a) mostra o número de impressões em cada um dos seis usuários, agrupadas por orientação política, e a Figura 3 (b) mostra o número total de publicações por orientação política. Essa Figura corrobora as observações anteriores: o viés das publicações reflete o viés dos usuários.

Cabe destacar que a grande diferença entre a ordem de aparição das fontes nas Figuras 1 e 1 é fruto da filtragem realizada pelo Facebook. Um dos objetivos do presente trabalho é propor um modelo analítico que nos permita compreender os efeitos de tal filtragem nas métricas de visibilidade e ocupação das fontes nas timelines.

Embora as observações apresentadas acima em parte sejam esperadas, cabe destacar que analisamos também usuários neutros. Para usuários neutros, que não curtem nenhuma fonte, seria de esperar que a presença de publicações fosse semelhante a Figura 1, ou então que existisse uma uniformidade entre as fontes. A Figura 2 mostra que esse não é o caso. Podemos constatar o alto número de impressões da fonte M5S no eleitor indeciso. É importante observar que o partido M5S foi o partido que recebeu mais votos nas eleições italianas. $\mathrm{O}$ modelo analítico apresentado a seguir nos permite realizar 


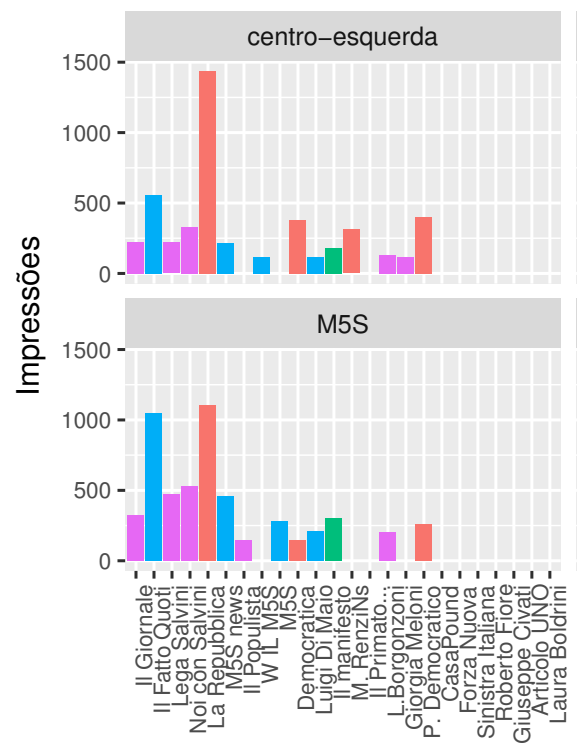

Orientação da Fonte

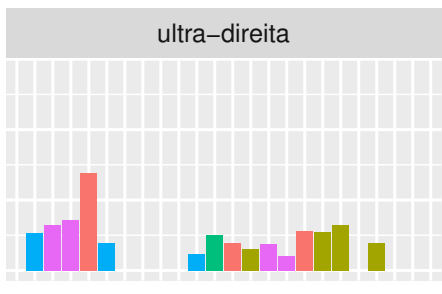

direita

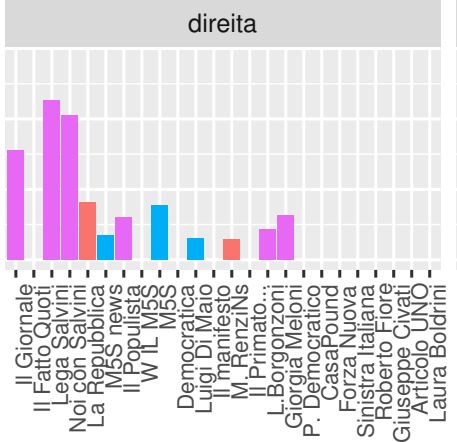

Fonte
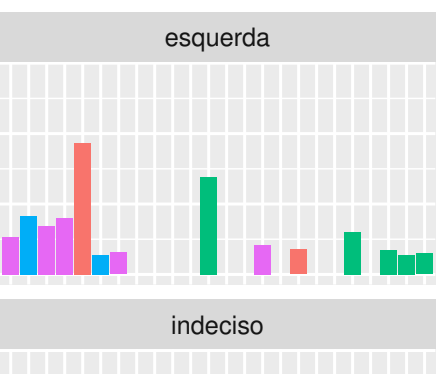

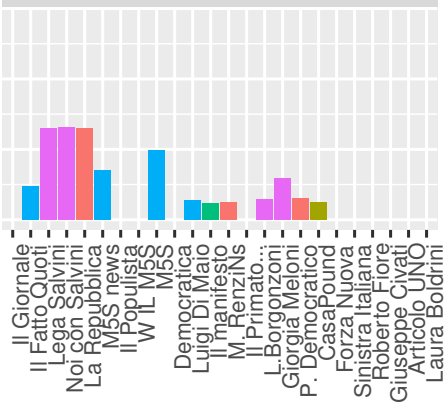

Zง

centro-esquerda

esquerda M5S direita

Figura 2. Total de impressões no topo da timeline por fonte em cada usuário (Apenas ocupações superiores a 0.2 estão representadas)

estudos contrafactuais, para averiguar o que ocorre com a visibilidade das fontes caso diferentes filtros sejam adotados.

\section{Uma visão geral sobre a modelagem de timelines}

A seguir, apresentamos intuitivamente as ideias que suportam o modelo analítico introduzido na seção seguinte.

\subsection{Analogias entre filas, caches e timelines}

Em redes sociais virtuais, as publicações criadas pelas fontes fluem através das conexões entre os seus respectivos membros e alcançam as timelines dos usuários interessados. Desta forma, timelines são um canal de comunicação entre fontes e usuários.

Na sua forma mais simples, as publicações são organizadas por ordem de chegada, de forma que estas entram e saem das timelines de acordo com uma ordem cronológica. Os algoritmos de personalização somente filtram as publicações da fonte $j$ na timeline do usuário $i$, transformando uma taxa de criação de conteúdo $\Lambda_{j}$ em uma taxa de exibição de mensagens $\lambda_{i j}$.

O comportamento recém descrito, é o comportamento de uma fila do tipo primeiro a entrar, primeiro a sair, ou, como é mais conhecida, como uma fila FIFO (first-in, first-out). Desta forma, acreditamos ser natural utilizar a teoria de filas para modelar as métricas de interesses das timelines.

Também argumentamos que existem inúmeras similaridades entre timelines e caches de conteúdos. Tanto timelines quanto caches são utilizadas para armazenar conteúdos de interesse a usuários. Ambas podem ser encaradas como filtros, tendo em 


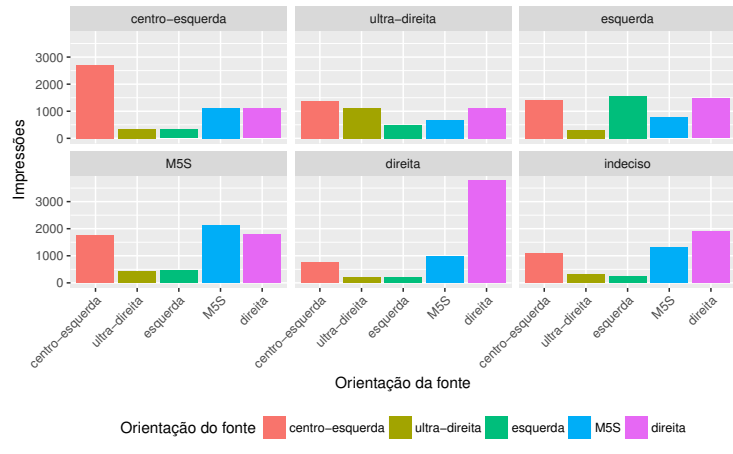

(a)

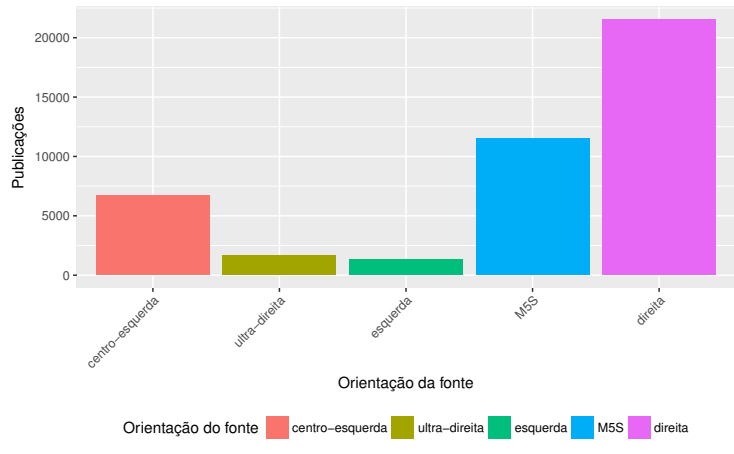

(b)

Figura 3. (a) Total de impressões no topo da timeline (por orientação política), (b) Total de publicações por orientação política

vista que tem tamanho limitado. Além disso, ambas em geral armazenam conteúdos mais recentes, e removem conteúdos que não são mais de interesse dos usuários. E, principalmente, ambas aumentam a eficiência da obtenção de informação do que está guardado nelas.

Existe uma ampla literatura sobre caches e teoria de filas. Estabelecendo a relação entre filas, caches e timelines, podemos nos aproveitar desta literatura para estudar timelines que, por serem mais recentes, receberam menos atenção da comunidade científica em comparação com caches e filas, que são melhor entendidas. No caminho oposto, acreditamos que resultados obtidos através dos estudos de timelines podem ser resultar em novas políticas de caching ou em novas formas de distribuição de conteúdo.

\subsection{Timelines são caches?}

Neste trabalho, um de nossos objetivos é compreender como projetar timelines (tendo em vista suas similaridades com caches) para melhor prover conteúdo de interesse dos usuários. Cabe destacar, entretanto, que existem importantes diferenças entre timelines e caches. Usuários de timelines estão tipicamente interessados numa classe de itens relacionados a uma certa categoria ou usuários. Esta é a principal diferença entre os dois. Por exemplo, um usuário deseja seguir as últimas notícias de seu jornal favorito. Esta trata-se de uma consulta flexível, em comparação com uma busca mais específica, por exemplo, a busca por um determinado episódio de uma série de televisão. A Tabela 1 ilustra algumas das diferenças entre caches e timelines.

Pelas razões acima, embora timelines e caches tenham muitas similaridades, elas necessitam de políticas de inserção e remoção de conteúdos distintas. Algoritmos de caching clássicos prestam-se a servir requisições por itens específicos. Algoritmos para timelines, em contrapartida, precisam lidar com a distribuição de conteúdo baseada em classes, como por exemplo, um tópico especifico ou uma fonte preferida. Por estes motivos, consideramos que timelines são caches orientados a fontes ou classes e divergindo dos caches tradicionais que são orientados à requisição.

\section{Um modelo analítico para timelines}

A seguir, apresentamos o modelo analítico proposto, seguido por sua validação usando dados das eleições italianas de 2018. 
Tabela 1. Comparação entre timelines e caches

\begin{tabular}{l||l|l}
\hline & Timelines & Caches \\
\hline \hline Evento de interesse & publicação de conteúdo & chegada de requisições \\
\hline Decisões de & tomadas após & tomadas depois \\
inserção e remoção & a publicação & de um miss \\
\hline Requisições & para classe de conteúdos & para conteúdos específicos \\
\hline Controle & de itens por & de itens \\
de ocupação & classe & específicos \\
\hline
\end{tabular}

\subsection{Descrição do modelo}

Nesta seção, descrevemos um modelo analítico para capturar a dinâmica das publicações em uma timeline.

Tabela 2. Tabela de notação

\begin{tabular}{ll}
\hline \hline Variável & descrição \\
\hline$K$ & número de posições de interesse no topo da timeline (top K) \\
$j$ & $j$-ésima fonte \\
$i$ & $i$-ésimo cliente (usuário) \\
$S_{i}$ & número de fotografias tiradas no usuário $i$ \\
$I_{i j}$ & número de impressões da fonte $j$ no usuário $i$ \\
$D_{i j}$ & número de publicações distintas da fonte $j$ visualizadas pelo usuário $i$ \\
$\Lambda_{j}$ & taxa de criação de publicações da fonte $j$ \\
$\lambda_{i j}$ & taxa efetiva de chegada de publicações de $j$ na timeline de $i$ \\
$\lambda_{i,-j}$ & taxa efetiva de chegada de publicações de outras fontes (que não $j$ ) na timeline de $i$ \\
$\lambda_{i}$ & taxa total de chegada na timeline de $i$ \\
$T_{i j}$ & valor esperado para tempo de permanência das publicações da fonte $j$ na timeline de $i$ \\
$\hat{\pi}_{i j}$ & visibilidade de $j$ medida na timeline de $i$ \\
$\pi_{i j}$ & visibilidade segundo modelo \\
$\hat{N}_{i j}$ & ocupação média das publicações da fonte $j$ medida na timeline de $i$ \\
$N_{i j}$ & ocupação média das publicações de $j$ medida na timeline de $i$ segundo modelo proposto \\
\hline
\end{tabular}

Dividimos os membros das redes sociais virtuais entre fontes e usuários. Membros que geram conteúdos são fontes, e membros que consomem tais conteúdos são os usuários. Ao administrar sua timeline, um usuário ou grupo de usuários pode subscrever a fontes, e um subconjunto das publicações destas fontes será exibida na timeline. Note que numa rede em que a maior parte dos conteúdos é gerada pelos próprios usuários (ex., Facebook), o papel de cada usuário dinamicamente muda entre fonte e usuário.

Seja $\mathcal{I}:=\{1, \ldots, i, \ldots I\}$ o conjunto de usuários em estudo, cada usuário associado a uma timeline, e seja $\mathcal{J}:=\{1, \ldots, j, \ldots J\}$ o conjunto de fontes. Seja $i$ o usuário de interesse, cuja timeline desejamos modelar (a notação é sumarizada na Tabela 2).

A fonte $j$ cria publicações segundo um processo Poisson com taxa $\Lambda_{j}$. Seja $\lambda_{i j} \leq$ $\Lambda_{j}$ a taxa efetiva com que a fonte $j$ alimenta a timeline $i$. A taxa agregada de publicações chegando na timeline do usuário $i$ é dada por

$$
\lambda_{i}=\sum_{j=1}^{J} \lambda_{i j}
$$




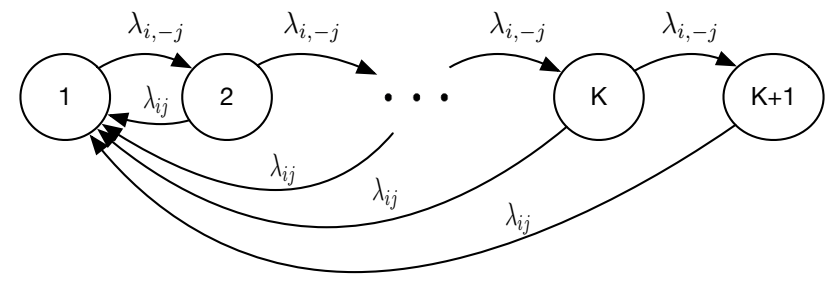

Figura 4. CTMC caracterizando a publicação da fonte $j$ na posição mais no topo na timeline do usuário $i$.

A taxa com que publicações de fontes, diferentes de $j$, chegam ao usuário $i$ é

$$
\lambda_{i,-j}=\lambda_{i}-\lambda_{i j}
$$

Dinâmica FIFO Assumimos que cada publicação entra no topo da timeline, na posição 1. Com taxa $\lambda_{i}$, as publicações tem sua posição incrementada em uma unidade. Estamos interessados nas primeiras $K$ posições (slots) da timeline. De forma equivalente, assumimos que a timeline tem tamanho $K$. Uma publicação é removida da timeline quando ela passa da posição $K$ para a posição fictícia $K+1$.

Cadeia de Markov A seguir, consideramos uma cadeia de Markov para capturar a evolução das publicações da fonte $j$, na timeline do usuário $i$. O objetivo é calcular a visibilidade e a ocupação de $j$ na timeline do usuário $i$. Por isso, não levamos em conta a posição de cada publicação de $j$ na timeline de $i$, mas apenas a posição da publicação mais no topo. Enquanto houver um post de $j$ na timeline de $i$, a fonte $j$ estará visível.

A Figura 4 mostra uma cadeia de Markov tempo contínuo (CMTC) cuja variável de estado $X$ representa a posição mais no topo ocupada por uma publicação da fonte $j$. Seja $\tilde{\pi}_{i j}(x)=P(X=x)$. Com taxa $\lambda_{i j}$, uma publicação de $j$ chega no topo timeline. Com taxa $\lambda_{i,-j}$, uma publicação de outra fonte faz com que as publicações de $j$ sejam movidas para a posição $\mathrm{k}+1$. A fonte $j$ estará fora da timeline se a sua publicação mais no topo encontrar-se na posição fictícia $K+1$. Logo, a visibilidade $\pi_{i j}$ é dada por

$$
\pi_{i j}=1-\tilde{\pi}_{i j}(K+1)
$$

Proposição 1. Em uma FIFO timeline, a visibilidade da fonte $j$ na timeline do usuário $i$ é

$$
\pi_{i j}=1-\left(\frac{\lambda_{i,-j}}{\lambda_{i}}\right)^{K}
$$

Demonstração. O resultado segue imediatamente a partir das equações de balanço do sistema, que podem ser derivadas diretamente a partir da Figura 4.

Proposição 2. Em uma FIFO timeline, a ocupação média da fonte $j$ na timeline do usuário i é

$$
N_{i j}=\frac{\lambda_{i j} K}{\lambda_{i}}
$$




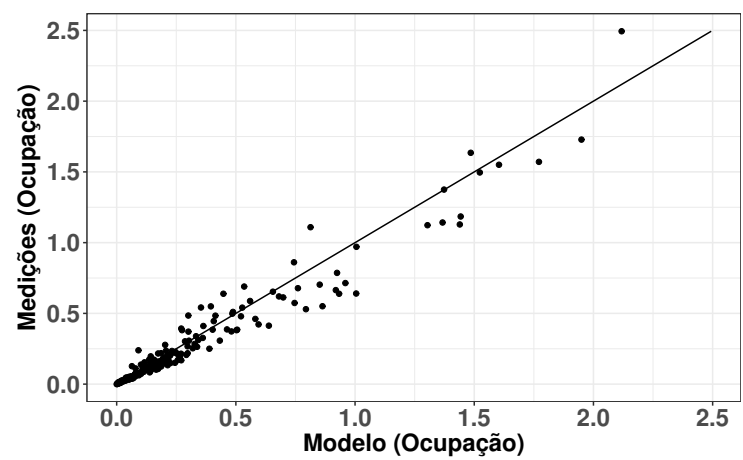

(a)

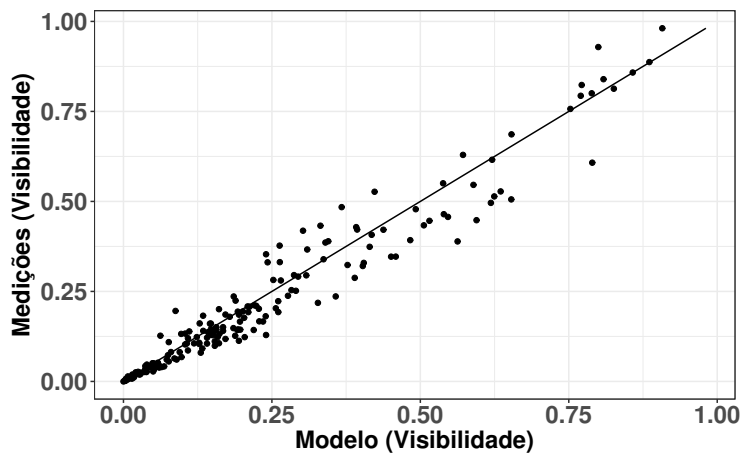

(b)

Figura 5. Validação do modelo: (a) Ocupação, (b) Visibilidade

Demonstração. Quando a $m$-ésima publicação entra na timeline ela fica visível até a chegada do $(m+K+1)$-ésima publicação. Já que o tempo médio entre chegadas é $1 / \lambda_{i}$, o tempo de residência da $m$-ésima publicação é $K / \lambda_{i}$. Em estado estacionário, seja $T_{i j} \mathrm{o}$ tempo médio que publicações de $j$ ficam na timeline de $i, T_{i j}=K / \lambda_{i}$. Pela lei de Little, $N_{i j}=\lambda_{i j} T_{i j}=\frac{\lambda_{i j} K}{\lambda_{i}}$.

Resumo modelo FIFO O modelo FIFO é um dos modelos analiticamente tratáveis mais simples que se possa conceber para uma timeline. Nesta seção, apresentamos o modelo FIFO e indicamos como ele pode ser usado para derivar a visibilidade e ocupação média de cada fonte em uma timeline. Em trabalhos futuros, pretendemos considerar modelos alternativos, mais flexíveis, como aqueles baseados em caches do tipo TTL.

\subsection{Validação}

A seguir, apresentamos a validação do modelo proposto. Nosso objetivo é indicar que o modelo tem capacidade expressiva para capturar os dados colhidos em ambiente real (eleições da Itália), e que ele é de fácil parametrização (requerendo apenas a taxa efetiva de publicações por fonte por usuário).

Chamamos de $D_{i j}$ o número de publicações distantas da fonte $j$ vistas pelo usuário $i$. Cabe ressaltar que esse número é menor ou igual a $I_{i j}$. A taxa $\lambda_{i j}$ é dada pela razão $D_{i j} / S_{j}$. Cada taxa obtida $\lambda_{i j}$ é substituída em (1) e em (2) para a obtenção da visibilidade, através da Proposição (1), e da ocupação, através da Proposição (4).

Cada ponto nas Figura 5 corresponde a um usuário e uma fonte. Um ponto $(x, y)$ indica que, para o usuário e a fonte em questão, o modelo prediz uma ocupação $x$ (respectivamente, visibilidade), e empiricamente observamos ocupação $y$ (resp., visibilidade). Os erros advém do fato, por exemplo, de nosso modelo assumir que as publicações nunca são reordenadas. Ainda assim, o fato de a maioria dos pontos estar próxima à reta $x=y$ ilustra o poder preditivo do modelo. O erro médio quadrático da ocupação foi igual a 0.0971 e o da visibilidade foi igual a 0.0527 .

\section{Análise contrafactual (what-if analysis)}

A seguir, combinamos o modelo proposto com os dados colhidos para realizar análise contrafactual de ocupações. Para tal, calculamos o viés entre as ocupações medidas em- 
piricamente e as ocupações preditas pelo modelo, usando a quantidade de publicações criadas por cada fonte (API do Facebook). O objetivo é comparar a ocupação após a filtragem do Facebook (ocupação medida empiricamente) com aquela que nosso modelo prediz como sendo a ocupação condizente com um sistema sem filtros (ocupação analítica obtida com o modelo proposto).

O viés é definido como a diferença $\hat{N}_{i j}-N_{j}$, onde $\hat{N}_{i j}$ é a ocupação média empírica da fonte $j$ na timeline $i$, e $N_{j}$ é dado por (4) e $\hat{N}_{i j}$ é dado por $I_{i j} / S_{i}$. Observe que o índice $i$ referente aos usuários é suprimido na expressão de $N_{j}$ pois na análise de ocupação ignorando filtros assumimos que a ocupação é igual para todos os usuários.

As Figuras 6 e 7 mostram que, de um modo geral, o viés positivo reflete a orientação dos usuários. No entanto, a fonte que mais produziu publicações, Il Giornale, foi penalizada em todos os usuários, e a segunda fonte que mais produziu, o Il Fatto Quotidiano só não foi penalizado no usuário que curtiu a sua página. O usuário neutro sofreu viés tanto positivo quanto negativo. A fonte M5S teve um forte viés positivo no usuário neutro. No entanto, o forte viés negativo do Il Fatto Quotidiano anulou esse efeito de forma que o viés total do M5S no usuário neutro foi negativo.

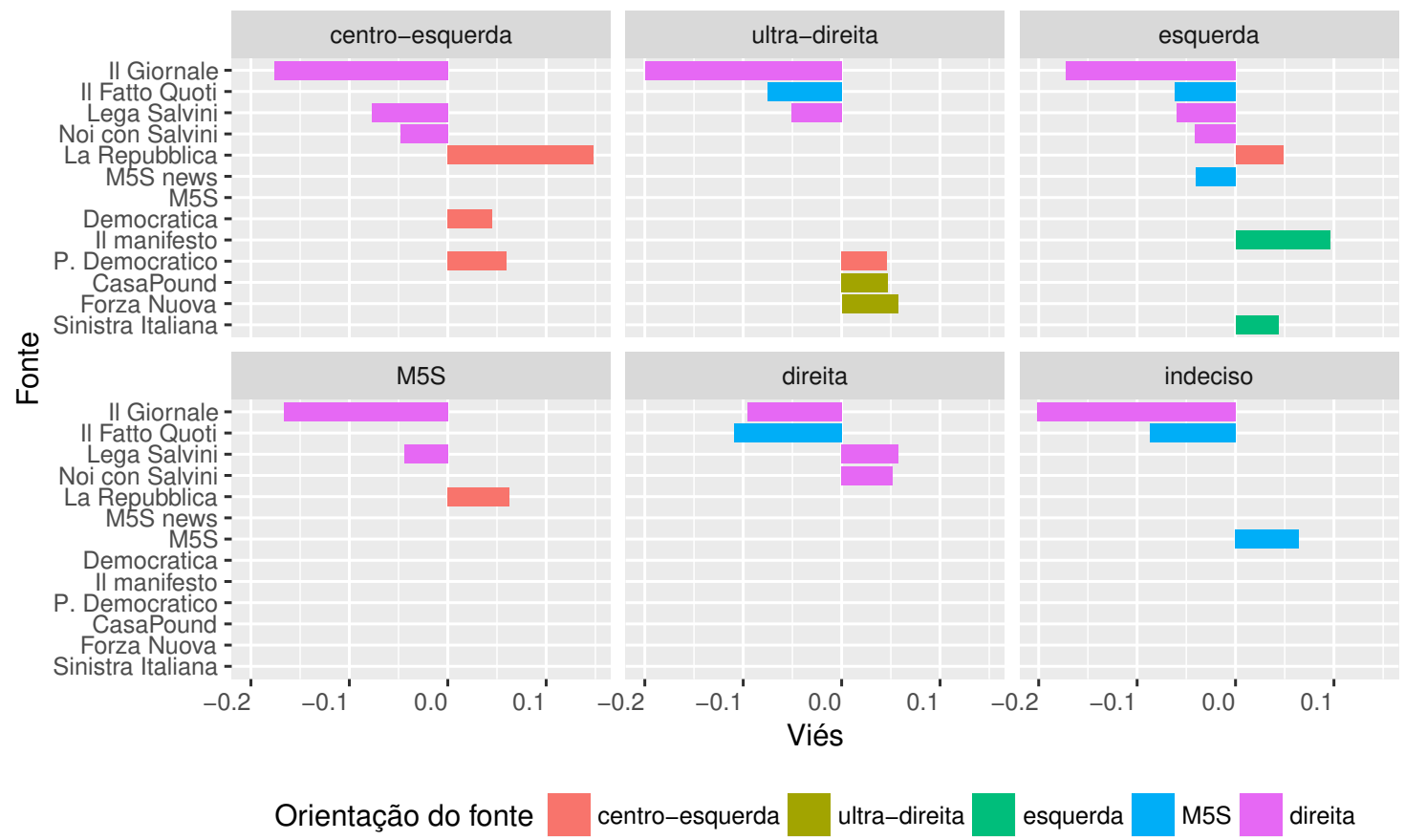

Figura 6. Viés por usuário e por fonte (estão representados apenas os casos em que o valor absoluto do viés foi maior do que $\mathbf{0 . 4 )}$

\section{Trabalhos relacionados}

O livro de [O'Neil 2016] mostrou diversas situações nas quais algoritmos podem reforçar preconceitos e tomar decisões que podem influenciar a sociedade como um todo. No contexto de mídias sociais e política, em [Epstein and Robertson 2015] foi demonstrado que manipulações em mecanismos de buscas são capazes de influenciar eleitores indecisos. Robôs fazendo propaganda política tentando influenciar eleições foram estudados no mundo todo em [Woolley and Howard 2017] e particularmente no Brasil em [Arnaudo 2017]. Um estudo realizado por [Eslami et al. 2015] mostrou que mais de 


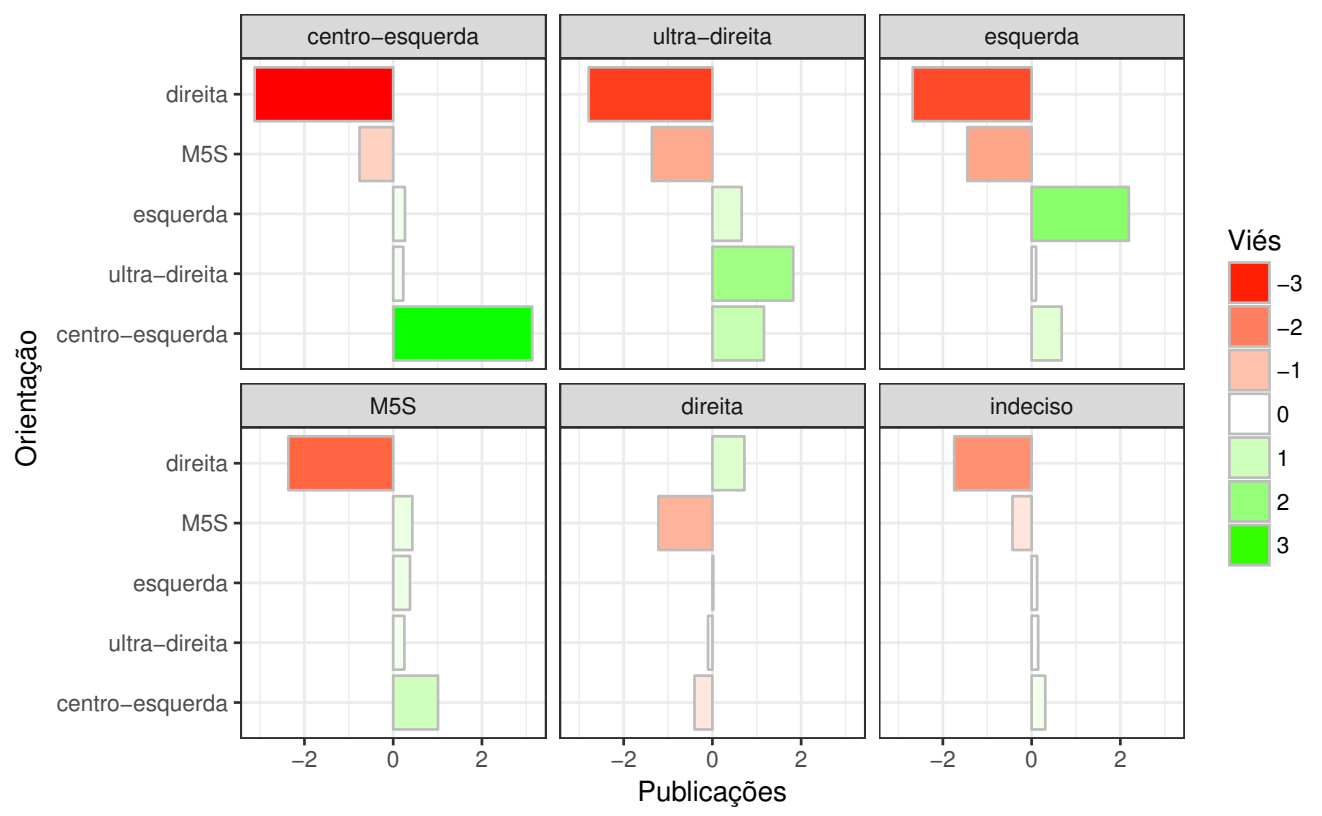

Figura 7. Viés por usuário e por orientação política

$60 \%$ das pessoas não tinham conhecimento da existência dos filtros de personalização do Facebook. Em [Bakshy et al. 2015] foi identificado que as escolhas individuais são o maior fator de influência sobre o nível de exposições a posições políticas opostas no Facebook. O nosso trabalho, por outro lado, mostrou que o algoritmo reforça as preferências dos usuários. Esse reforço de preferências pode ajudar a criar as filter bubbles identificadas em [Pariser 2011].

Neste trabalho, mostramos como modelos de caches e filas podem ser usados no estudo de timelines. Existe uma vasta literatura sobre ambos [Martina et al. 2014, Dehghan et al. 2016, Harchol-Balter 2013]. Acreditamos que esta conexão aqui estabelecida permita estender-se resultados destes domínios para as timelines.

Existe uma vasta literatura focada em fazer engenharia reversa dos algoritmos por trás de timelines [Andreou et al. 2018]. Entretanto, a literatura de modelos analíticos nessa área é bem mais escassa. O trabalho de [Altman et al. 2013] foi o primeiro a modelar uma timeline com um fila Fifo. O nosso trabalho propôs uma solução do sistema capaz de obter além da visibilidade, a ocupação das fontes. Em particular, não é de nosso conhecimento nenhum trabalho anterior que tenha feito uso de medições reais de timelines, conectando tais medições com modelos analíticos.

\section{Conclusões}

O Facebook afeta milhões de usuários da Internet todos os dias, e qualquer decisão algorítmica sobre as timelines pode ter importantes impactos sociais e políticos. Neste trabalho, propusemos uma metodologia envolvendo medições e um modelo analítico para quantificar métricas de ocupação, visibilidade e viés em timelines. Indicamos que o modelo proposto tem poder preditivo, e que permite fazer análise contrafactual de dados. Acreditamos que este seja um importante passo no sentido de garantir maior transparência para os usuários ao torná-los mais informados sobre os processos de filtragem aos quais as publicações por eles visualizadas estão submetidas. 
Agradecimentos Este projeto foi em parte conduzido pelo time associado do projeto THANES, com recursos do INRIA (França) e da FAPERJ (UFRJ/Brasil), tendo sido parcialmente financiado também pelo CNPq, CAPES e FAPESP.

\section{Referências}

Altman, E., Kumar, P., Venkatramanan, S., and Kumar, A. (2013). Competition over timeline in social networks. ASONAM, pages 1352-1357.

Andreou, A., Venkatadri, G., Goga, O., Gummadi, K. P., Loiseau, P., and Mislove, A. (2018). Investigating ad transparency mechanisms in social media. NDSS.

Arnaudo, D. (2017). Computational Propaganda in Brazil : Social Bots during Elections.

Bakshy, E., Messing, S., and Adamic, L. A. (2015). Exposure to ideologically diverse news and opinion on Facebook. Science, 348(6239):1130-1132.

Dehghan, M., Massoulie, L., Towsley, D., Menasché, D., and Tay, Y. C. (2016). A utility optimization approach to network cache design. In Proceedings - IEEE INFOCOM, volume 2016-July, pages 1-10.

Epstein, R. and Robertson, R. E. (2015). The search engine manipulation effect and its possible impact on the outcomes of elections. Nat. Academy of Sciences of the United States of America, 112(33):E4512-21.

Eslami, M., Rickman, A., Vaccaro, K., Aleyasen, A., Vuong, A., Karahalios, K., Hamilton, K., and Sandvig, C. (2015). I always assumed that I wasn't really that close to [her]. Human Factors in Computing (HCI), pages 153-162.

Facebook (2018a). Graph api reference /user-id/home.

Facebook (2018b). Log de alterações da graph api. Retrieved April 6, 2018 from: https://developers. facebook.com/docs/graph-api/changelog.

Harchol-Balter, M. (2013). Performance Modeling and Design of Computer Systems. Cambridge University Press, Cambridge.

Martina, V., Garetto, M., and Leonardi, E. (2014). A unified approach to the performance analysis of caching systems. IEEE INFOCOM, pages 2040-2048.

O’Neil, C. (2016). Weapons of Math Destruction: How Big Data Increases Inequality and Threatens Democracy. Crown Publishing Group, New York, NY, USA.

Pariser, E. (2011). The Filter Bubble: How the New Personalized Web Is Changing What We Read and How We Think. Penguin Publishing Group.

TechCruch (2016). How facebook news feed works. Retrieved December 31, 2017 from: https://techcrunch.com/2016/09/06/ ultimate-guide-to-the-news-feed/.

Tsaparas, P. (2017). Online social networks and media. http://www.cs.uoi.gr/ $\sim$ tsap/teaching/cs-114/references.html.

Woolley, S. C. and Howard, P. N. (2017). Computational propaganda worldwide: executive summary. 